\title{
Can Wind Lidars Measure Turbulence?
}

\author{
A. SATHE \\ Faculty of Aerospace Engineering, Section Wind Energy, Delft University of Technology, Delft, Netherlands \\ J. Mann, J. GotTschall, And M. S. Courtney \\ Wind Energy Division, Risø DTU, Roskilde, Denmark
}

(Manuscript received 5 October 2010, in final form 10 January 2011)

\begin{abstract}
Modeling of the systematic errors in the second-order moments of wind speeds measured by continuouswave (ZephIR) and pulsed (WindCube) lidars is presented. These lidars use the conical scanning technique to measure the velocity field. The model captures the effect of volume illumination and conical scanning. The predictions are compared with the measurements from the ZephIR, WindCube, and sonic anemometers at a flat terrain test site under different atmospheric stability conditions. The sonic measurements are used at several heights on a meteorological mast in combination with lidars that are placed on the ground. Results show that the systematic errors are up to $90 \%$ for the vertical velocity variance, whereas they are up to $70 \%$ for the horizontal velocity variance. For the ZephIR, the systematic errors increase with height, whereas for the WindCube, they decrease with height. The systematic errors also vary with atmospheric stability and are low for unstable conditions. In general, for both lidars, the model agrees well with the measurements at all heights and under different atmospheric stability conditions. For the ZephIR, the model results are improved when an additional low-pass filter for the 3-s scan is also modeled. It is concluded that with the current measurement configuration, these lidars cannot be used to measure turbulence precisely.
\end{abstract}

\section{Introduction}

A theoretical model is developed to estimate the systematic errors in the second-order moments of wind speeds in the atmospheric surface layer measured by lidars. The systematic errors are those that arise resulting from the averaging effect in the sample or pulse volume and the relatively large circle in which Doppler lidars scan to obtain two-component horizontal wind profiles. Two types of lidars are considered, the ZephIR, developed by QinetiQ (Natural Power), as a continuous-wave (CW) lidar and the WindCube, developed by Leosphere as a pulsed lidar. ${ }^{1}$ The verification is carried out by comparing the variances measured by the ZephIR and WindCube

\footnotetext{
${ }^{1}$ These lidars are not the second versions, WindCubeV2 and ZephIR 300, which were released in 2010, but the first versions of the instruments.

Corresponding author address: A. Sathe, L \& R, Section Wind Energy, TU Delft, Kluyverweg 1, 2629 HS Delft, Netherlands. E-mail: a.r.sathe@tudelft.nl
}

with that of the sonic anemometers placed at different heights on a meteorological mast.

Wind energy has expanded rapidly for several decades and every year thousands of multimegawatt wind turbines are being installed all over the world. The importance of wind speed measurements can never be overstated because the power produced from the wind turbine is directly proportional to the cube of the wind speed, at least below turbine-rated wind speeds. Atmospheric turbulence is one of the main inputs in assessing loads on the wind turbines. Thus, accurate estimation of wind speed and turbulence at several heights is crucial for the successful development of a wind farm. In wind energy, the current standard is the use of meteorological masts equipped with cup and/or sonic anemometers. However, tall meteorological masts are very expensive, and offshore the costs increase significantly. The advent of remote sensing devices like lidars gives a further boost to the development of wind energy. In recent years with the introduction of the ZephIR and WindCube, there has been a surge in the verification campaigns of comparing the lidar mean wind speed with that of a cup anemometer for wind energy applications (Smith et al. 
2006; Kindler et al. 2007; Courtney et al. 2008; Peña et al. 2009). Courtney et al. (2008) discuss the advantages and disadvantages of CW and pulsed lidars. To use a lidar as a standard measuring instrument in the future, for example, in the International Electrotechnical Commission (IEC) standards for loads (IEC 2005a,b) and power performance measurements (IEC 2005c), a fair degree of confidence is also required in the turbulence measurements.

Although lidars have been introduced in wind energy recently, for meteorology they have been investigated previously to measure turbulence using different scanning techniques. One of the first remote sensing (Doppler radar) turbulence studies using a full $360^{\circ}$ scan in a horizontal plane was carried out by Browning and Wexler (1968), where the limitations of horizontal homogeneity and vertical wind shear are explained in detail. Wilson (1970) modified the technique from Browning and Wexler (1968) and performed turbulence measurements over snow. Kropfli (1986) extended the technique to accommodate turbulence scales of motion larger than those described in Wilson (1970) and showed that these techniques could be used to make reasonable estimates of turbulent kinetic energy and momentum flux by modeling the random errors in the measurements.

Eberhard et al. (1989) studied turbulence using Doppler lidar and modeled the random errors using a partial Fourier decomposition method, which gave better estimates of the errors than Wilson (1970) and Kropfli (1986). Gal-Chen et al. (1992) presented a technique for analyzing lidar data for turbulence measurements using the scans at two levels, and produced estimates of fluxes in the mixed layer and spectra of the horizontal velocity at the surface. Banakh et al. (1995) presented an analysis of estimating the random errors in the measurement of the mean wind speed by lidars using the theory of isotropic turbulence. Banta et al. (2002) studied the turbulence characteristics under the conditions of low-level jets, using the vertical slice scans of radial velocities. Smalikho et al. (2005) presented a method to use lidar data for the estimation of turbulent energy dissipation rate to study wake vortices of an aircraft. A comprehensive review is given in Engelbart et al. (2007), which covers different remote sensing techniques for turbulence measurements, including lidars. A review of the use of lidars for wind energy applications is also presented in Emeis et al. (2007). Pichugina et al. (2008) demonstrated the sensitivity of the streamwise velocity variance to the spatial and temporal averaging, also by using the technique of vertical slice scans of radial velocities. Recently, studies have been carried out to model the spatial averaging effects (Sjöholm et al. 2009) and compare the 3D turbulence measurements using three staring lidars (Mann et al. 2009). Wagner et al. (2009) modeled the systematic errors by approximating the conical scan and the scan time as a length scale, providing first estimates of the variances of the longitudinal component of wind velocity. Mann et al. (2010) estimated the momentum fluxes using lidars and modeled the unfiltered turbulence from the $\mathrm{CW}$ lidar, where the model compares reasonably well with the measurements. In the present work, line-of-sight averaging and the full extent of conical scanning is considered. An additional low-pass filter for the 3-s scan is also considered for the ZephIR.

In the remaining sections, the work is described in detail. Section 2 describes the theory, where the systematic error in the second-order moments is modeled for the ZephIR and WindCube. Section 3 provides details of the measurements used for comparison with the model. Section 4 describes the results along with some inferences. Section 5 gives a discussion on the systematic errors of the second-order moments, while section 6 provides a conclusion.

\section{Theory}

The model in this study is developed for the conical scanning and velocity-azimuth display (VAD) technique of data processing. The approach is similar to Wyngaard (1968) and Citriniti and George (1997), where turbulence measured by the hot-wire anemometer probe was modeled.

Figure 1 shows the lidar emitting the laser beam at different azimuth angles $\theta$. The azimuth angles increase from $0^{\circ}$ to $360^{\circ}$ in the clockwise direction, as for the geographical convention. The line-of-sight velocity (also called radial velocity $v_{r}$ ) is measured by the lidar at each azimuth angle. The half-opening angle $\phi\left(=90^{\circ}-\right.$ elevation angle) is kept constant throughout the scan. The CW and pulsed lidars work on the principle of backscattering of the emitted radiation, and the subsequent detection of the Doppler shift in the frequency of the received radiation. The Doppler shift in the frequency is related to $v_{r}$ by

$$
\delta f=2 \frac{v_{r}}{\lambda},
$$

where $f$ and $\lambda$ are the frequency and wavelength of the emitted radiation. Mathematically, $v_{r}$ is given as the dot product of the unit directional vector and the velocity field at the point of focus for a CW lidar, and the center of the range gate (Lindelöw 2007) for the pulsed lidar,

$$
v_{r}(\theta)=\mathbf{n}(\theta) \cdot \mathbf{v}\left[d_{f} \mathbf{n}(\theta)\right]
$$

where $d_{f}$ is the focus distance for the CW lidar or the distance to the center of the range gate for the pulsed lidar at which the wind speeds are measured, $\mathbf{v}=(u, v, w)$ 


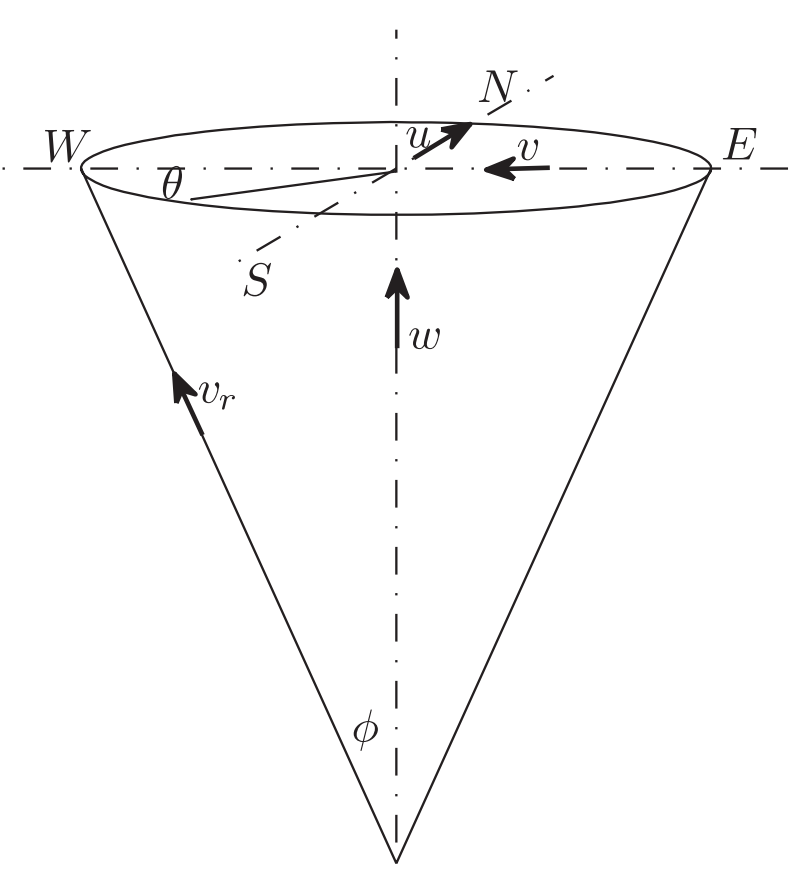

FIG. 1. Schematic of the velocity-azimuth display scanning.

is the instantaneous velocity field evaluated at the focus point or the center of the range gate $d_{f} \mathbf{n}(\theta)$, and $\mathbf{n}(\theta)$ is the unit directional vector given as

$$
\mathbf{n}(\theta)=(\cos \theta \sin \phi, \sin \theta \sin \phi, \cos \phi) .
$$

In practice it is impossible to obtain the backscattered radiation precisely from only the focus point, and there is always backscattered radiation of different intensities from different regions in space along the line of sight. Hence, it is necessary to assign appropriate weights to the backscattered intensity such that the weight corresponding to the focus point or the center of the range gate is the highest. Mathematically, the weighted average radial velocity can be written as

$$
\tilde{v}_{r}(\theta)=\int_{-\infty}^{\infty} \varphi(s) \mathbf{n}(\theta) \cdot \mathbf{v}\left[s \mathbf{n}(\theta)+d_{f} \mathbf{n}(\theta)\right] d s
$$

where $\varphi(s)$ is any weighting function, integrating to one, and $s$ is the distance along the beam from the focus. For simplicity we assume that $s=0$ corresponds to the focus distance.

Following are the main assumptions of our model:

(i) The terrain is homogeneous.

(ii) The flow field is frozen during the scan.

(iii) Equation (4) with an appropriately chosen $\varphi(s)$ models the averaging well.

(iv) The spatial structure of the turbulent flow is described well by the spectral tensor model of Mann (1994).

\section{a. Systematic turbulence errors for the ZephIR lidar}

The ZephIR transmits the laser beam through a constantly rotating prism, giving the required half-opening angle of nominally $30^{\circ}$. Each of up to five heights is scanned for 1 or $3 \mathrm{~s}$, corresponding to one or three complete rotations of the prism. The beam is then refocused to the next height in the sequence and the scanning procedure is repeated. Up to five different heights can be selected, with the sequence (with five heights and 3-s scans) taking up to $18 \mathrm{~s}$ to complete. Thus, the lidar spends less than $20 \%$ of the time required to make a wind profile on any one of the five heights. A typical scan at each height consists of 50 measurements of $v_{r}$ on the azimuth circle. If we assume the coordinate system such that $u$ is aligned to the mean wind direction, $v$ is perpendicular to the mean wind direction, $w$ is the vertical component, and the mean wind comes from the north, then $\tilde{v}_{r}(\theta)$ can be expressed as

$$
\tilde{v}_{r}(\theta)=A+B \cos \theta+C \sin \theta,
$$

where the coefficients $A=w_{q q} \cos \phi, B=u_{q q} \sin \phi$, and $C=v_{q q} \sin \phi$, and the sign ambiguity in $\tilde{v}_{r}(\theta)$ is neglected (see Mann et al. 2010). We use the subscript $q q$ to denote the velocity components measured by ZephIR, because they are not the true velocity components $u, v$, and $w$. The assumption that the mean wind comes from the north is only made for simplicity. For a lidar measuring at many points on the azimuth circle the choice of the mean wind direction does not matter since averaging over the entire circle is carried out. The values of the coefficients $A, B$, and $C$ are found using the least squares method by fitting Eq. (5) to the measured values of $\tilde{v}_{r}(\theta)$ at all scanned azimuth angles. The coefficients can be written as Fourier integrals,

$$
\begin{aligned}
& A=\frac{1}{2 \pi} \int_{0}^{2 \pi} \tilde{v}_{r}(\theta) d \theta, \\
& B=\frac{1}{\pi} \int_{0}^{2 \pi} \tilde{v}_{r}(\theta) \cos \theta d \theta, \\
& C=\frac{1}{\pi} \int_{0}^{2 \pi} \tilde{v}_{r}(\theta) \sin \theta d \theta .
\end{aligned}
$$

We proceed by deriving expressions for the $w_{q q}$ variance. The expressions for the (co)variances of the remaining components of wind velocity can be derived in a similar manner.

The variance of $A$ is defined as $\sigma_{A}^{2}=\left\langle A^{\prime 2}\right\rangle$, where the angle brackets denotes ensemble averaging of a variable. From the above definition of $A$, we can write

$$
\sigma_{A}^{2}=\left\langle w_{q q}^{\prime 2}\right\rangle \cos ^{2} \phi
$$


Using Eq. (6) we can also write,

$$
\sigma_{A}^{2}=\left\langle\left[\frac{1}{2 \pi} \int_{0}^{2 \pi} \tilde{v}_{r}^{\prime}(\theta) d \theta\right]^{2}\right\rangle
$$

Substituting $\tilde{v}_{r}(\theta)$ from Eq. (4) into Eq. (10), converting the square of the integral into a double integral, and interchanging the order of integration and averaging we get,

$$
\begin{aligned}
\sigma_{A}^{2} & =\frac{1}{4 \pi^{2}} \int_{0}^{2 \pi} \int_{0}^{2 \pi} \int_{-\infty}^{\infty} \int_{-\infty}^{\infty}\left\langle\mathrm{v}_{i}^{\prime}\left[s_{1} \mathbf{n}\left(\theta_{1}\right)+d_{f} \mathbf{n}\left(\theta_{1}\right)\right] \mathbf{v}_{j}^{\prime}\left[s_{2} \mathbf{n}\left(\theta_{2}\right)+d_{f} \mathbf{n}\left(\theta_{2}\right)\right]\right\rangle \varphi\left(s_{1}\right) \varphi\left(s_{2}\right) n_{i}\left(\theta_{1}\right) n_{j}\left(\theta_{2}\right) d s_{1} d s_{2} d \theta_{1} d \theta_{2}, \\
& =\frac{1}{4 \pi^{2}} \int_{0}^{2 \pi} \int_{0}^{2 \pi} \int_{-\infty}^{\infty} \int_{-\infty}^{\infty} R_{i j}(\mathbf{r}) \varphi\left(s_{1}\right) \varphi\left(s_{2}\right) n_{i}\left(\theta_{1}\right) n_{j}\left(\theta_{2}\right) d s_{1} d s_{2} d \theta_{1} d \theta_{2},
\end{aligned}
$$

where $\left\langle\mathrm{v}_{i}^{\prime}\left[s_{1} \mathbf{n}\left(\theta_{1}\right)+d_{f} \mathbf{n}\left(\theta_{1}\right)\right] \mathrm{v}_{j}^{\prime}\left[s_{2} \mathbf{n}\left(\theta_{2}\right)+d_{f} \mathbf{n}\left(\theta_{2}\right)\right]\right\rangle=R_{i j}(\boldsymbol{r})$ is the covariance tensor separated by a distance $\mathbf{r}=$ $\left[s_{1} \mathbf{n}\left(\theta_{1}\right)+d_{f} \mathbf{n}\left(\theta_{1}\right)\right]-\left[s_{2} \mathbf{n}\left(\theta_{2}\right)+d_{f} \mathbf{n}\left(\theta_{2}\right)\right]$ and is related to the three-dimensional spectral velocity tensor $\Phi_{i j}(\mathbf{k})$ by the inverse Fourier transform,

$$
R_{i j}(\mathbf{r})=\int \Phi_{i j}(\mathbf{k}) e^{i \mathbf{k} \cdot \mathbf{r}} d \mathbf{k}
$$

where $\int d \mathbf{k} \equiv \int_{-\infty}^{\infty} \int_{-\infty}^{\infty} \int_{-\infty}^{\infty} d k_{1} d k_{2} d k_{3}, \mathbf{k}=\left(k_{1}, k_{2}, k_{3}\right)$ denotes the wave vector and the subscripts $i, j$ take the values from 1 to 3. Inserting Eq. (12) into Eq. (11) we get,

$$
\sigma_{A}^{2}=\int \Phi_{i j}(\mathbf{k})\left\{\int_{-\infty}^{\infty} \varphi\left(s_{1}\right)\left[\frac{1}{2 \pi} \int_{0}^{2 \pi} n_{i}\left(\theta_{1}\right) e^{i\left(s_{1}+d_{f}\right) \mathbf{k} \cdot \mathbf{n}\left(\theta_{1}\right)} d \theta_{1}\right] d s_{1}\right\}\left\{\int_{-\infty}^{\infty} \phi\left(s_{2}\right)\left[\frac{1}{2 \pi} \int_{0}^{2 \pi} n_{j}\left(\theta_{2}\right) e^{i\left(s_{2}+d_{f}\right) \mathbf{k} \cdot \mathbf{n}\left(\theta_{2}\right)} d \theta_{2}\right] d s_{2}\right\} d \mathbf{k} .
$$

Let $\alpha_{i}(\mathbf{k})=\int_{-\infty}^{\infty} \varphi(s)\left[1 / 2 \pi \int_{0}^{2 \pi} n_{i}(\theta) e^{i\left(s+d_{f}\right) \mathbf{k} \cdot \mathbf{n}\left(\theta_{1}\right)} d \theta\right] d s$, which physically represents the line-of-sight and conical averaging. Equation (13) can then be written as (using Eq. 9),

$$
\left\langle w_{q q}^{\prime 2}\right\rangle \cos ^{2} \phi=\int \Phi_{i j}(\mathbf{k}) \alpha_{i}(\mathbf{k}) \alpha_{j}^{*}(\mathbf{k}) d \mathbf{k}
$$

where the asterisk denotes complex conjugation. Thus the integral reduces to evaluating $\alpha_{i}(\mathbf{k})$, since the analytical expressions for $\Phi_{i j}(\mathbf{k})$ are given in Mann (1994). Equation (16) can then be estimated numerically. For a CW lidar, $\varphi(s)$ is well approximated by a Lorentzian function (Sonnenschein and Horrigan 1971),

$$
\varphi(s)=\frac{1}{\pi} \frac{l}{l^{2}+s^{2}},
$$

where $l$ is the Rayleigh length $\left(=\lambda_{b} d_{f}^{2} / \pi r_{b}^{2}\right.$, where $\lambda_{b}=$ $1.55 \mu \mathrm{m}$ is the wavelength of the emitted radiation, and $r_{b}=19.5 \mathrm{~mm}$ is the beam radius). An attempt has been made to obtain analytical expressions for $\alpha_{i}(\mathbf{k})$. However, no general analytical solution exists for $\alpha_{i}(\mathbf{k})$ and at most the integral can be reduced (by integrating over $s$ ) to

$$
\alpha_{i}(\mathbf{k})=\frac{1}{2 \pi} e^{i d_{f} k_{3} \cos \phi} \int_{0}^{2 \pi} n_{i}\left(\theta+\theta_{0}\right) e^{i d_{f} k_{h} \sin \phi \cos \theta} e^{-l\left|k_{h} \cos \theta \sin \phi+k_{3} \cos \phi\right|} d \theta
$$

where $k_{h}=\sqrt{k_{1}^{2}+k_{2}^{2}}$ is the magnitude of the horizontal wave vector, $\cos \theta_{0}=k_{1} / k_{h}, \sin \theta_{0}=k_{2} / k_{h}$, and $n_{i}\left(\theta+\theta_{0}\right)$ is the component of the unit directional vector obtained from Eq. (3). Thus numerical integration has to be applied also for the evaluation of $\alpha_{i}(\mathbf{k})$.

A similar approach is taken for deriving $u_{q q}$ and $v_{q q}$ variances, where we obtain,

$$
\begin{aligned}
& \left\langle u_{q q}^{\prime 2}\right\rangle \sin ^{2} \phi=\int \Phi_{i j}(\mathbf{k}) \beta_{i}(\boldsymbol{k}) \beta_{j}^{*}(\mathbf{k}) d \mathbf{k}, \\
& \left\langle v_{q q}^{\prime 2}\right\rangle \sin ^{2} \phi=\int \Phi_{i j}(\mathbf{k}) \gamma_{i}(\mathbf{k}) \gamma_{j}^{*}(\mathbf{k}) d \mathbf{k} .
\end{aligned}
$$

The corresponding $\beta$ and $\gamma$ functions are

$$
\beta_{i}(\mathbf{k})=\frac{1}{\pi} e^{i d_{f} k_{3} \cos \phi} \int_{0}^{2 \pi} n_{i}\left(\theta+\theta_{0}\right) \cos \left(\theta+\theta_{0}\right) e^{i d_{f} k_{h} \sin \phi \cos \theta} e^{-l\left|k_{h} \cos \theta \sin \phi+k_{3} \cos \phi\right|} d \theta
$$




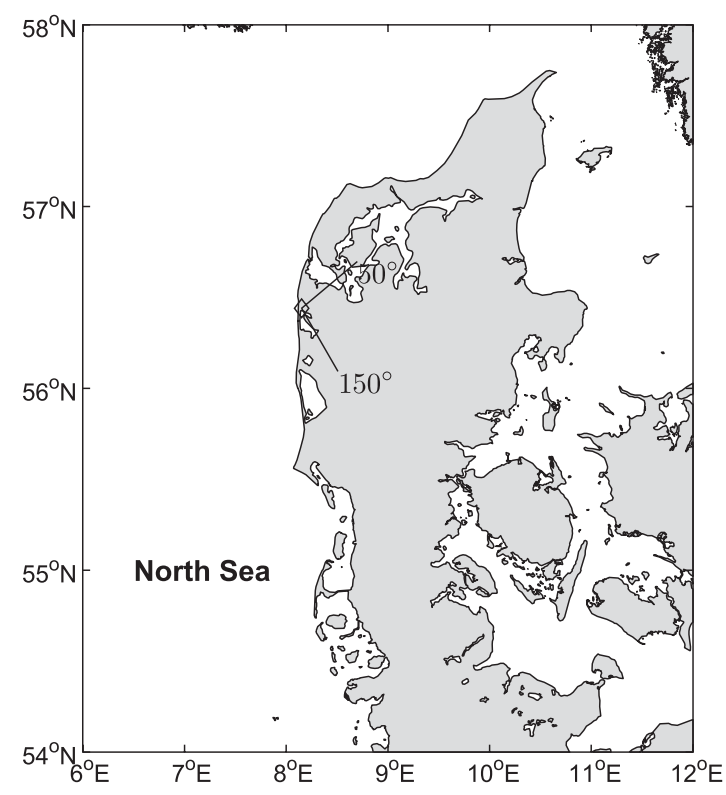

(a)

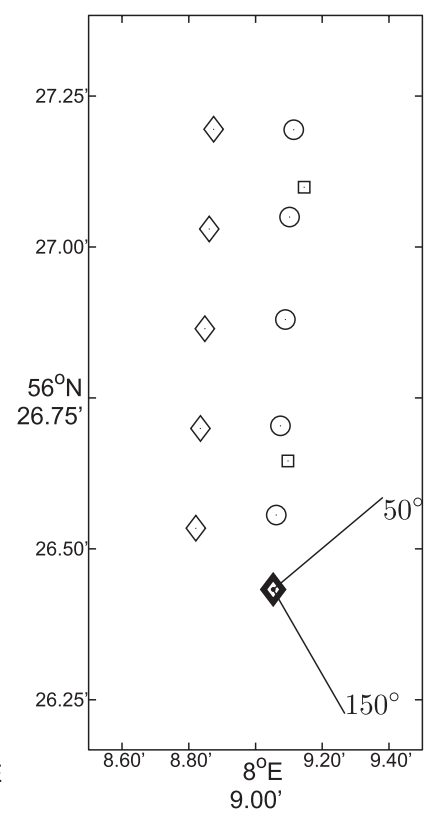

(b)

FIG. 2. Location of the Høvsøre meteorological mast and details of the site. The wind turbines (circles), light tower (squares), and the meteorological masts (diamonds) are shown. The meteorological mast from which the measurements are used is indicated (dark diamond) and the selected wind directions are also shown.

$$
\gamma_{i}(\mathbf{k})=\frac{1}{\pi} e^{i d_{f} k_{3} \cos \phi} \int_{0}^{2 \pi} n_{i}\left(\theta+\theta_{0}\right) \sin \left(\theta+\theta_{0}\right) e^{i d_{f} k_{h} \sin \phi \cos \theta} e^{-l\left|k_{h} \cos \theta \sin \phi+k_{3} \cos \phi\right|} d \theta .
$$

The derivation of the covariances is merely a combination of the weighting functions $\alpha_{i}(\mathbf{k}), \beta_{i}(\mathbf{k})$, and $\gamma_{i}(\mathbf{k})$, and their complex conjugates used with $\Phi_{i j}(\mathbf{k})$.

\section{MODELING THE LOW-PASS FILTERING EFFECT RESULTING FROM THE 3-S SCAN}

Because the ZephIR scans three circles in approximately $3 \mathrm{~s}$, there will be a low-pass filter effect in turbulence measurements. We assume a length scale $L_{f}=$ $\langle u\rangle \times 3$ s such that it represents the 3 -s averaging. We assume that the ZephIR scans a circle infinitely fast for $3 \mathrm{~s}$. We model the corresponding filtering effect by a simple rectangular filter, such that,

$$
f(x)=\left\{\begin{aligned}
\frac{1}{L_{f}} & \text { for } \quad|x|<\frac{L_{f}}{2} \\
0 & \text { elsewhere }
\end{aligned}\right.
$$

where $x$ is the center of the scanning circle and $f(x)$ is any function of $x$. The corresponding spectral transfer function is given as

$$
\hat{T}_{f}\left(k_{1}\right)=\operatorname{sinc}^{2}\left(\frac{k_{1} L_{f}}{2}\right),
$$

where $\operatorname{sinc}(x)=\sin (x) / x$. The variances of $u_{q q}, v_{q q}$, and $w_{q q}$ are given as

$$
\begin{aligned}
\left\langle u_{q q}^{\prime 2}\right\rangle \sin ^{2} \phi & =\int \Phi_{i j}(\mathbf{k}) \beta_{i}(\mathbf{k}) \beta_{j}^{*}(\mathbf{k}) \hat{T}_{f}\left(k_{1}\right) d \mathbf{k}, \\
\left\langle v_{q q}^{\prime 2}\right\rangle \sin ^{2} \phi & =\int \Phi_{i j}(\mathbf{k}) \gamma_{i}(\mathbf{k}) \gamma_{j}^{*}(\mathbf{k}) \hat{T}_{f}\left(k_{1}\right) d \mathbf{k}, \\
\left\langle w_{q q}^{\prime 2}\right\rangle \cos ^{2} \phi & =\int \Phi_{i j}(\mathbf{k}) \alpha_{i}(\mathbf{k}) \alpha_{j}^{*}(\mathbf{k}) \hat{T}_{f}\left(k_{1}\right) d \mathbf{k} .
\end{aligned}
$$

\section{b. Systematic turbulence errors for the WindCube lidar}

The assumption made in section $2 \mathrm{a}$ that the mean wind direction comes from the north cannot be made for the WindCube, because it measures at four azimuth 


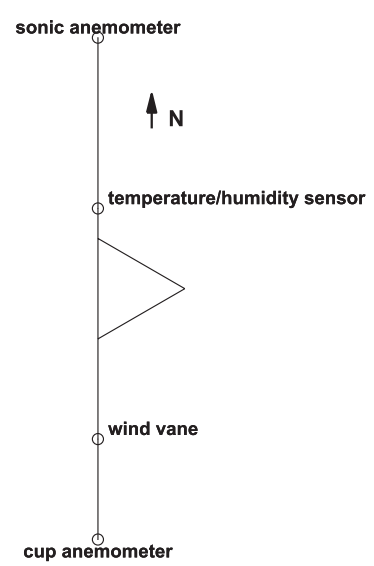

(a) Sensor Orientation

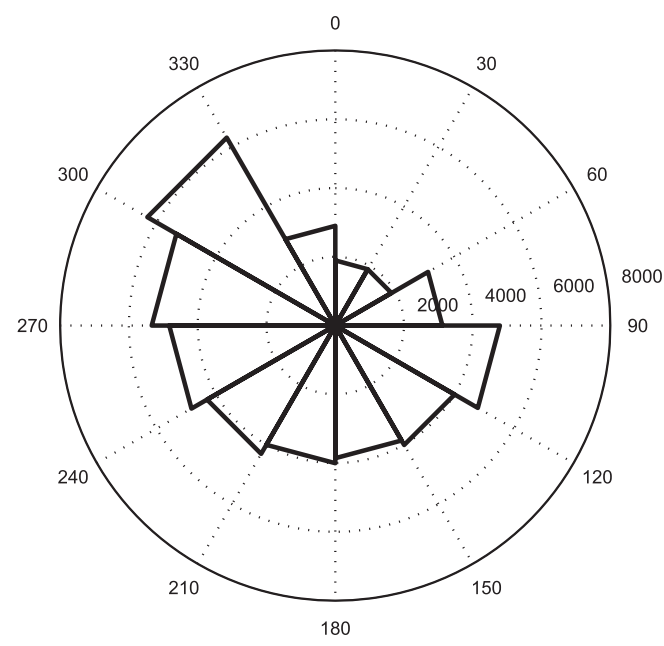

(b) Wind Rose

FIG. 3. Orientation of the sensors on the meteorological mast and wind rose at $60 \mathrm{~m}$. The numbers inside the circles are the number of 10-min observations.

angles only (cf. Fig. 1), for example, north, east, south and west. In this case the coordinate system is such that $u$ is aligned in the mean wind direction. Thus,

$$
\begin{aligned}
& u_{\mathrm{wc}}=u_{N S} \cos \Theta+u_{E W} \sin \Theta, \\
& v_{\mathrm{wc}}=u_{N S} \sin \Theta-u_{E W} \cos \Theta,
\end{aligned}
$$

where $u_{N S}$ and $u_{E W}$ denote wind speeds in the northsouth and east-west directions, respectively, $\Theta$ denotes the wind direction, and the subscript wc denotes the velocity components measured by WindCube. From simple geometrical considerations (cf. Fig. 1),

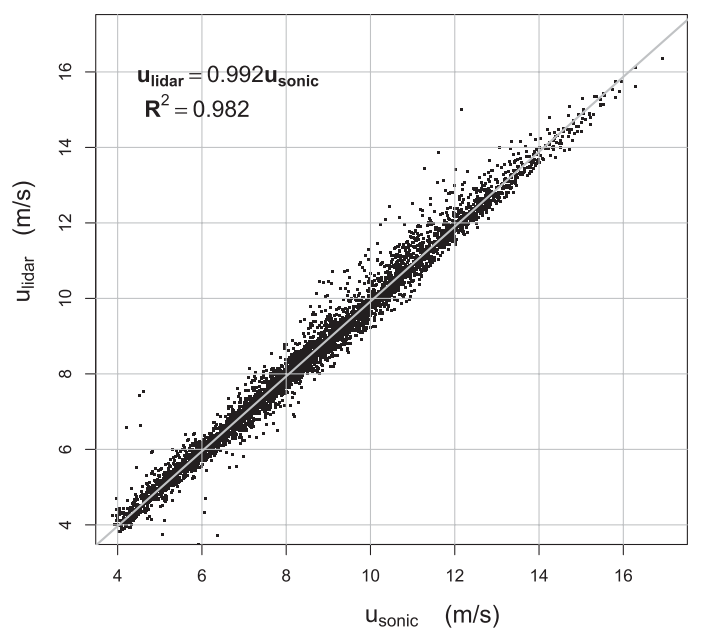

(a) ZephIR

$$
\begin{gathered}
u_{N S}=\frac{\tilde{v}_{r N}-\tilde{v}_{r S}}{2 \sin \phi}, \\
u_{E W}=\frac{\tilde{v}_{r E}-\tilde{v}_{r W}}{2 \sin \phi},
\end{gathered}
$$

where $\tilde{v}_{r N}, \tilde{v}_{r S}, \tilde{v}_{r E}$, and $\tilde{v}_{r W}$ are the weighted average radial velocities in the north, south, east and west directions, respectively. For the $w$ component,

$$
w_{\mathrm{wc}}=\frac{P\left(\tilde{v}_{r N}+\tilde{v}_{r S}\right)+Q\left(\tilde{v}_{r E}+\tilde{v}_{r W}\right)}{2 \cos \phi},
$$

where $P$ and $Q$ are the weights associated with the wind direction such that $P+Q=1$. Leosphere uses

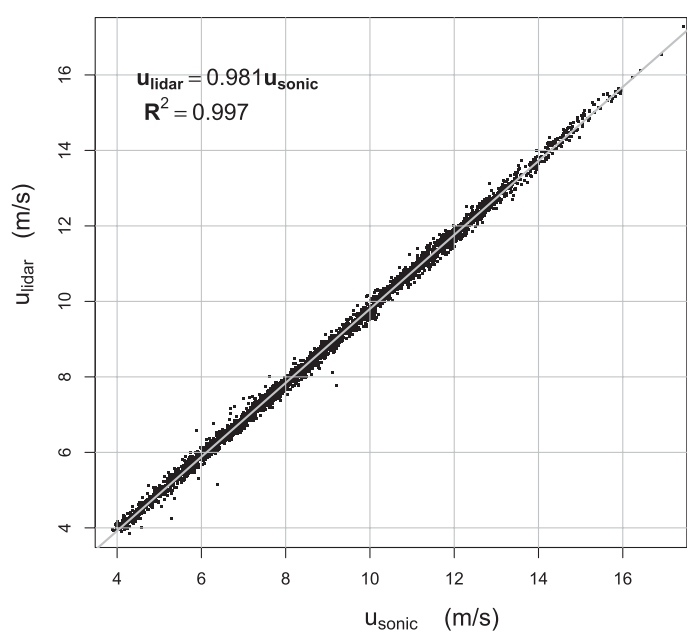

(b) WindCube

FIG. 4. Comparison of the mean wind speed measured by (left) lidars and (right) sonic anemometer. 
$P=\cos ^{2} \Theta$ and $Q=\sin ^{2} \Theta$, and hence, we use the same in our calculations.

We proceed by deriving expressions for the $u_{\mathrm{wc}}$ variance. The expressions for the (co)variances of the remaining components of wind velocity can be derived in a similar manner. Substituting Eqs. (28) and (29) into Eq. (26), we get

$$
u_{\mathrm{wc}}=\frac{1}{2 \sin \phi}\left[\left(\tilde{v}_{r N}-\tilde{v}_{r S}\right) \cos \Theta+\left(\tilde{v}_{r E}-\tilde{v}_{r W}\right) \sin \Theta\right] .
$$

We define unit vectors in the four directions as

$$
\begin{aligned}
\mathbf{n}_{N} & =\mathbf{n}(-\Theta), \\
\mathbf{n}_{S} & =\mathbf{n}(\pi-\Theta), \\
\mathbf{n}_{E} & =\mathbf{n}\left(\frac{\pi}{2}-\Theta\right), \\
\mathbf{n}_{W} & =\mathbf{n}\left(\frac{3 \pi}{2}-\Theta\right),
\end{aligned}
$$

where $\mathbf{n}_{N}, \mathbf{n}_{S}, \mathbf{n}_{E}$, and $\mathbf{n}_{W}$ are the unit directional vectors in the north, south, east, and west directions, respectively. From Eq. (4), for the north direction,

$$
\tilde{v}_{r N}=\int_{-\infty}^{\infty} \varphi(s) \mathbf{n}_{N} \cdot \mathbf{v}\left(s \mathbf{n}_{N}+d_{f} \mathbf{n}_{N}\right) d s .
$$

To further simplify the notation we define the translation operator $T_{\delta}$ acting on any scalar or vector field $\xi(\mathbf{x})$,

$$
T_{\delta} \xi(\mathbf{x})=\xi(\mathbf{x}+\delta)
$$

We also define a convolution operator $C_{\mathbf{n}}$ acting on any scalar or vector field as

$$
C_{\mathbf{n}} \mathbf{v}(\mathbf{x})=\int_{-\infty}^{\infty} \varphi(s) \mathbf{n} \cdot \mathbf{v}(\mathbf{x}+\mathbf{n} s) d s
$$

For the north direction, Eq. (33) can be written as

$$
\tilde{v}_{r N}=C_{\mathbf{n}_{N}} T_{d_{f} \mathbf{n}_{N}} \mathbf{v}
$$

TABLE 1. Classification of atmospheric stability according to Monin-Obukhov length intervals

\begin{tabular}{ll}
\hline \hline Very stable (vs) & $10 \leq L_{\mathrm{MO}} \leq 50 \mathrm{~m}$ \\
Stable (s) & $50 \leq L_{\mathrm{MO}} \leq 200 \mathrm{~m}$ \\
Near-neutral stable (nns) & $200 \leq L_{\mathrm{MO}} \leq 500 \mathrm{~m}$ \\
Neutral (n) & $\left|L_{\mathrm{MO}}\right| \geq 50 \mathrm{~m}$ \\
Near-neutral unstable (nnu) & $-500 \leq L_{\mathrm{MO}} \leq-200 \mathrm{~m}$ \\
Unstable (u) & $-200 \leq L_{\mathrm{MO}} \leq-100 \mathrm{~m}$ \\
Very unstable (vu) & $-100 \leq L_{\mathrm{MO}} \leq-50 \mathrm{~m}$ \\
\hline
\end{tabular}

We get similar expressions for the south, east, and west directions. Equation (31) can then be written as

$$
\begin{aligned}
u_{\mathrm{wc}}= & \frac{1}{2 \sin \phi}\left[\left(C_{\mathbf{n}_{N}} T_{d_{f} \mathbf{n}_{N}}-C_{n_{S}} T_{d_{f} \mathbf{n}_{S}}\right) \cos \Theta\right. \\
& \left.+\left(C_{\mathbf{n}_{E}} T_{d_{f} \mathbf{n}_{E}}-C_{\mathbf{n}_{W}} T_{d_{f} \mathbf{n}_{W}}\right) \sin \Theta\right] \mathbf{v}
\end{aligned}
$$

We also know that, by definition,

$$
\left\langle u^{\prime 2}\right\rangle=\int\left\langle\hat{u}(\mathbf{k}) \hat{u}^{*}(\mathbf{k})\right\rangle d \mathbf{k},
$$

where ${ }^{\wedge}$ denotes the Fourier transform and $*$ denotes complex conjugation. In the Fourier space we have

$$
\begin{aligned}
& \widehat{T_{\delta} \mathbf{v}}(\mathbf{k})=e^{i \mathbf{k} \cdot \delta} \hat{\mathbf{v}}(\mathbf{k}), \\
& \widehat{C_{n} \mathbf{v}}(\mathbf{k})=\hat{\varphi}(\mathbf{n} \cdot \mathbf{k}) \mathbf{n} \cdot \hat{\mathbf{v}}(\mathbf{k}),
\end{aligned}
$$

where $\hat{\phi}(k)=\operatorname{sinc}^{2}\left(k l_{p} / 2\right)$, considering that the weighting function for a pulsed lidar is commonly defined as

$$
\varphi(s)= \begin{cases}\frac{l_{p}-|s|}{l_{p}^{2}} & \text { for }|s|<l_{p} \\ 0 & \text { elsewhere }\end{cases}
$$

where $l_{p}$ is the half-length of the ideally rectangular light pulse leaving the lidar, assuming the matching time windowing ( $=2 l_{p} / c$, where $c$ is the speed of light). In reality, the weighting function may be more rounded, but this will not affect the conclusions that are drawn. Thus, in Fourier space Eq. (37) can then be written as

$$
\hat{u}_{\mathrm{wc}}(\mathbf{k})=\frac{1}{2 \sin \phi}\left\{\begin{array}{l}
{\left[\mathbf{k}_{N} e^{i d_{f} \mathbf{k} \cdot \mathbf{n}_{N}} \operatorname{sinc}^{2}\left(\mathbf{k} \cdot \mathbf{n}_{N} l_{p} / 2\right)-\mathbf{n}_{S} e^{i d_{f} \mathbf{k} \cdot \mathbf{n}_{S}} \operatorname{sinc}^{2}\left(\mathbf{k} \cdot \mathbf{n}_{S} l_{p} / 2\right)\right] \cos \Theta} \\
{\left[\mathbf{n}_{E} e^{i d_{f} \mathbf{k} \cdot \mathbf{n}_{E}} \operatorname{sinc}^{2}\left(\mathbf{k} \cdot \mathbf{n}_{E} l_{p} / 2\right)-\mathbf{n}_{W} e^{i d_{f} \mathbf{k} \cdot \mathbf{n}_{W}} \operatorname{sinc}^{2}\left(\mathbf{k} \cdot \mathbf{n}_{W} l_{p} / 2\right)\right] \sin \Theta}
\end{array}\right\} \cdot \hat{\mathbf{v}}(\mathbf{k}) \equiv \mathbf{b}(\mathbf{k}) \cdot \hat{\mathbf{v}}(\mathbf{k}),
$$

and the variance [from Eq. (38)] as

$$
\left\langle u_{\mathrm{wc}}^{\prime 2}\right\rangle=\int \Phi_{i j}(\mathbf{k}) b_{i}(\mathbf{k}) b_{j}^{*}(\mathbf{k}) d \mathbf{k},
$$

where we have implicitly used the relation $\Phi_{i j}(\mathbf{k})=$ $\left\langle\hat{\mathrm{v}}_{i}(\mathbf{k}) \hat{\mathrm{v}}_{j}^{*}(\mathbf{k})\right\rangle$. The (co)variances of other components can be estimated in a similar manner by first estimating the 


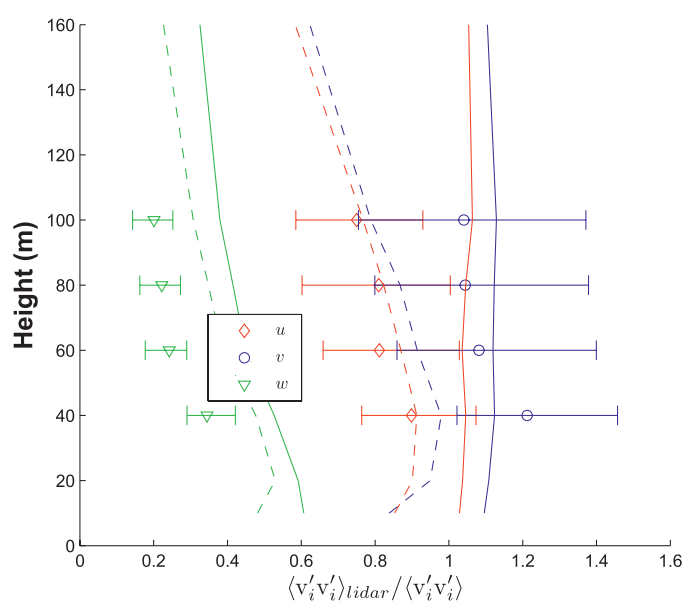

(a) very unstable

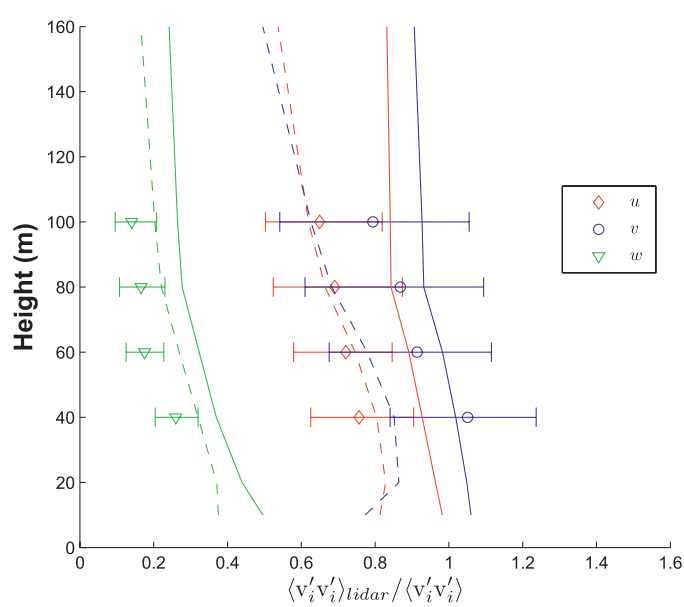

(c) near neutral unstable

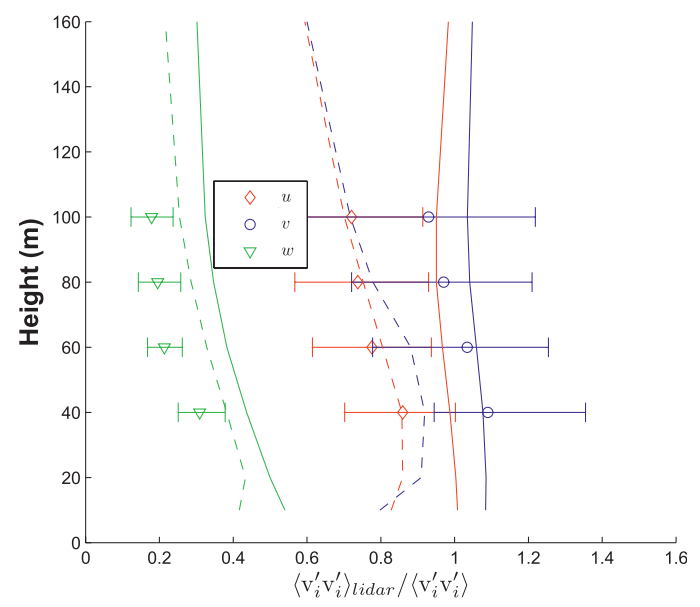

(b) unstable

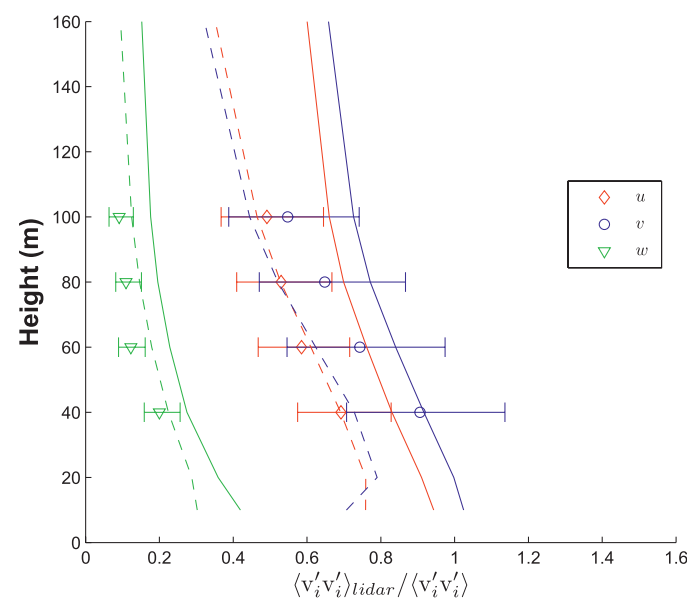

(d) neutral

FIG. 5. (a)-(g) ZephIR systematic errors under different atmospheric stability conditions in the eastern sector ranging from very unstable to very stable. The symbols indicate measurements. The solid lines are the theory without the low-pass filter, and the dashed lines are with the low-pass filter. The colored symbols represent the $u$ (red diamonds), $v$ (blue circles), and $w$ (green inverted triangles). The horizontal lines represent the error bars in the first and third quartile range.

corresponding weighting functions $c_{i}(\mathbf{k})$ and $a_{i}(\mathbf{k})$ for the $v_{\mathrm{wc}}$ and $w_{\mathrm{wc}}$ components, respectively.

\section{Description of the measurements}

The measurements were performed at the Danish National Test Center for Large Wind Turbines at Høvsøre, Denmark. Figure 2 shows the layout of the test center and the location of the used reference meteorological mast, a 116.5-m-tall, intensively equipped mast located at the coordinates $56^{\circ} 26^{\prime} 26^{\prime \prime} \mathrm{N}, 08^{\circ} 09^{\prime} 03^{\prime \prime} \mathrm{E}$, (indicated by a dark diamond in Fig. 2b). The site is about $2 \mathrm{~km}$ from the west coast of Denmark. The eastern sector is characterized by flat homogeneous terrain, and to the south is a lagoon.

Our reference measurements for this study are the sonic anemometer measurements taken at 40,60, 80 , and $100 \mathrm{~m}$. The measured three-dimensional wind speeds are resolved with a frequency of $20 \mathrm{~Hz}$ and then reduced to the respective 10-min statistics (mean values and standard deviations or variances). All sonic anemometers are placed on the north booms of the meteorological mast (Fig. 3a), resulting in unusable data when the wind is from the south resulting from the wake of the mast. In combination with the sonic measurements, wind speeds from a ZephIR (coordinates $56^{\circ} 26^{\prime} 26.9556^{\prime \prime} \mathrm{N}, 08^{\circ} 09^{\prime} 2.448^{\prime \prime} \mathrm{E}$ ) and a WindCube (coordinates $56^{\circ} 26^{\prime} 26.0556^{\prime \prime} \mathrm{N}, 0.8^{\circ} 09^{\prime} 3.226^{\prime \prime} \mathrm{E}$ ) are used. The ZephIR is located about $35 \mathrm{~m}$ north of the meteorological mast and the WindCube is located about $11 \mathrm{~m}$ northwest of the meteorological mast. Reference and lidar data were collected over two different time periods: for the WindCube between January and April 2009, and for the ZephIR between April and November 2009. To further avoid the influence of the wakes from the wind turbines and the meteorological mast on 


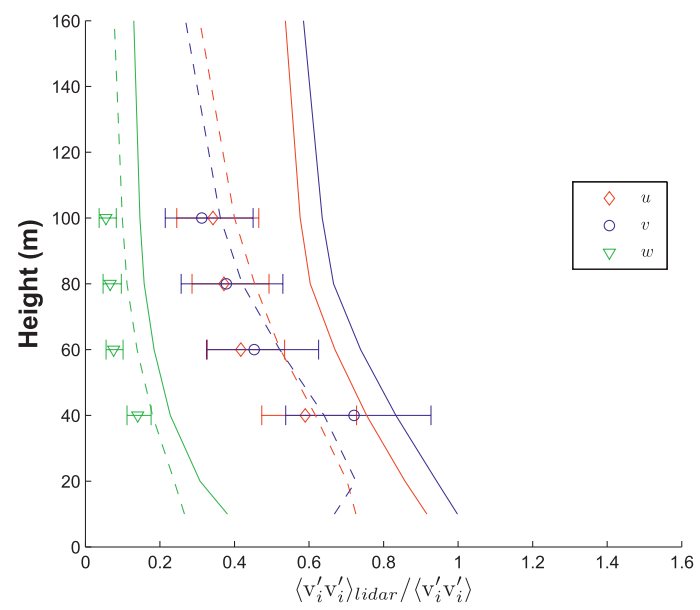

(e) near neutral stable

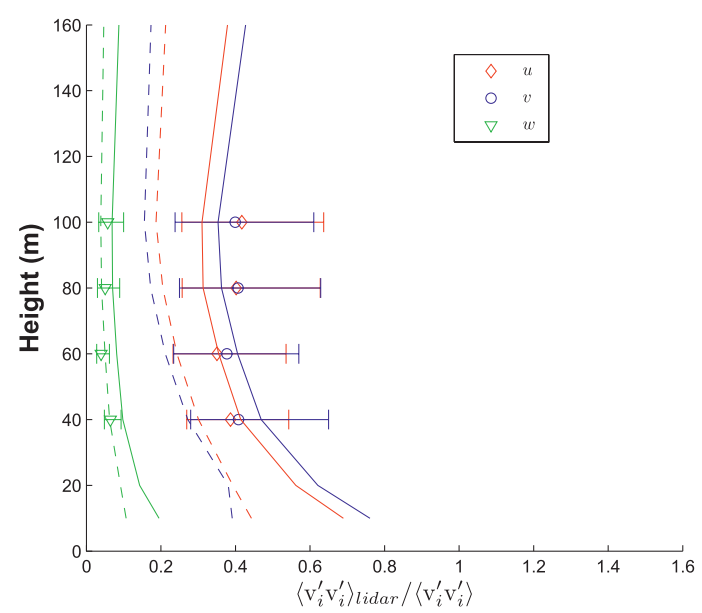

(g) very stable

lidar measurements, and inhomogeneities resulting from the sudden change of roughness (the sea-land transition; see Fig. $2 \mathrm{a})$, only data periods with easterly winds $\left(50^{\circ}-\right.$ $150^{\circ}$ ) are analyzed. Figure $3 \mathrm{~b}$ shows that although the dominant wind direction is west-northwest, there is also sufficient data in the chosen directional (eastern) sector. For the ZephIR 5530 data points were used after using the entire filter, whereas for the WindCube 4003 data points were used.

The precision of the sonic anemometer measurements is estimated to be about $\pm 1.5 \%$. From comparisons with cup anemometers, the mean error of the WindCube in typical flat coastal conditions is within $\pm 0.05 \mathrm{~m} \mathrm{~s}^{-1}$, with a standard deviation in mixed shear conditions of about $0.15 \mathrm{~m} \mathrm{~s}^{-1}$. The corresponding uncertainty for the measurements made with a ZephIR is slightly higher [a detailed list of different error sources is given in Lindelöw-Marsden (2007)].

Figure 4 shows the comparison of the 10-min mean horizontal wind speed (at $100 \mathrm{~m}$ ) measured by the

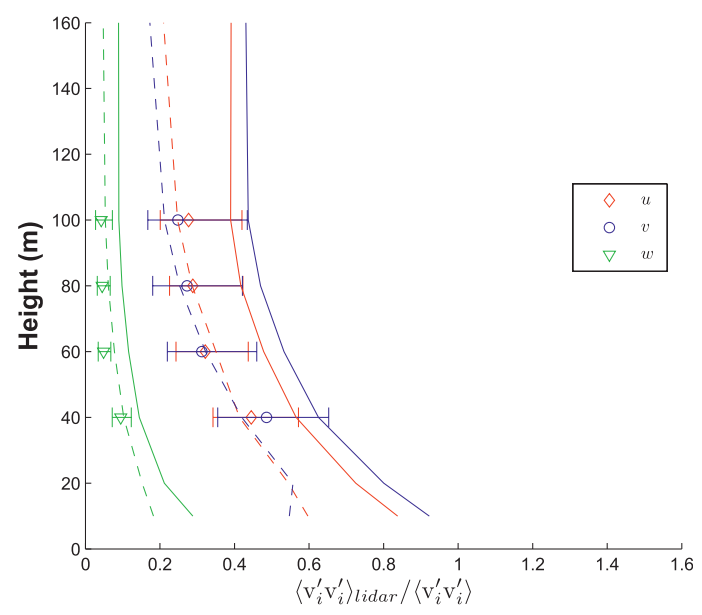

(f) stable

FIG. 5. (Continued)

ZephIR and WindCube with the sonic anemometer. Data are shown for the easterly winds $\left(50^{\circ}-150^{\circ}\right)$ and reference mean wind speeds between 4 and $25 \mathrm{~m} \mathrm{~s}^{-1}$. To guarantee repeatable conditions the data were furthermore filtered with respect to rain (i.e., only 10-min periods with no precipitation were considered) and the availability of the lidar (i.e., $100 \%$ of the fast data within a 10-min period had to be available). The lidar observations agree reasonably well with those of the sonic anemometer, with coefficients of determination $R^{2}>0.98$, where the data of the WindCube shows a significantly better correlation than those of the ZephIR.

\section{Comparison of models with the measurements}

The estimation of $\Phi_{i j}$ using the model from Mann (1994) requires three input parameters: $\alpha \epsilon^{2 / 3}$, which is a product of the spectral Kolmogorov constant $\alpha$ (Monin and Yaglom 1975) and the rate of viscous dissipation of specific turbulent kinetic energy $\epsilon^{2 / 3}$; a length scale $L$, and an 
anisotropy parameter $\Gamma$. We use these input parameters obtained by fitting the sonic anemometer measurements under different atmospheric stability conditions at several heights on the meteorological mast in the eastern sector (Peña et al. 2010). The classification of atmospheric stability (Table 1) is based on the Monin-Obukhov length $\left(L_{\mathrm{MO}}\right)$ intervals (Gryning et al. 2007).

Here, $L_{\mathrm{MO}}$ is estimated using the eddy covariance method (Kaimal and Finnigan 1994) from the high-frequency $(20 \mathrm{~Hz})$ measurements at $20 \mathrm{~m}$. Mathematically, $L_{\mathrm{MO}}$ is given as

$$
L_{\mathrm{MO}}=-\frac{u_{*}^{3} T}{\kappa g \overline{w^{\prime} \theta_{v}^{\prime}}},
$$

where $u_{*}$ is the friction velocity, $\kappa=0.4$ is the von Kármán constant, $g$ is the acceleration resulting from gravity, $T$ is the absolute temperature, $\theta_{v}$ is the virtual potential temperature, and $\overline{w^{\prime} \theta_{v}^{\prime}}$ (covariance of $w$ and $\theta_{v}$ ) is the virtual kinematic heat flux; $u_{*}$ is estimated as

$$
u_{*}=\sqrt[4]{\overline{u^{\prime} w^{\prime}}+\overline{v^{\prime} w^{\prime}}}
$$

where $\overline{u^{\prime} w^{\prime}}$ (covariance of $u$ and $w$ ) and $\overline{v^{\prime} w^{\prime}}$ (covariance of $v$ and $w$ ) are the vertical fluxes of the horizontal momentum.

\section{a. Definition of the systematic error}

For simplicity we define systematic error as the ratio of the lidar to the true second-order moment. Thus, a ratio equal to one would signify no systematic error, whereas deviations from unity signify systematic error. By definition, the true second-order moment of a velocity component is given as

$$
\left\langle\mathrm{v}_{j}^{\prime} \mathrm{v}_{j}^{\prime}\right\rangle=\int \Phi_{i j}(\mathbf{k}) d \mathbf{k}
$$

The theoretical systematic errors are calculated by taking the ratio of lidar second-order moments [Eqs. (14), (17), (18), and (42)] to the true second-order moment [Eq. (46)]. The numerical integration is carried out using an adaptive algorithm (Genz and Malik 1980). For experimental comparison, the second-order moments measured by sonic anemometers are considered to be true second-order moments. Thus, experimentally, the systematic errors are estimated by taking the ratio of the measured lidar second-order moments to sonic secondorder moments.

\section{b. Comparison with the ZephIR measurements}

Figure 5 shows the comparison of the modeled and measured systematic errors for $u, v$, and $w$ variances

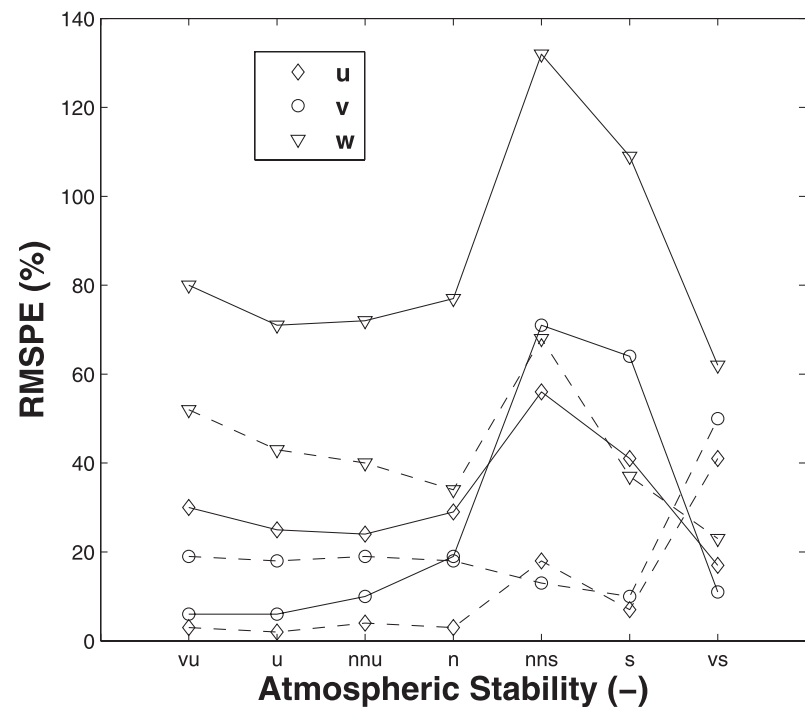

FIG. 6. Root-mean-square percent error (RMSPE) in the prediction of the systematic errors for the ZephIR. The model without the low-pass filter (solid line) and the model with the low-pass filter (dashed line) are shown. See Table 1 for the meaning of the abbreviations on the $x$ axis. Symbols as in Fig. 5 .

over 10-min periods. The theoretical points are shown both with and without the low-pass filter. For the lowpass filter, the model is dependent on the mean wind speed and the plots are shown for $\langle u\rangle=9 \mathrm{~m} \mathrm{~s}^{-1}$ at all heights, because this is the mean wind speed at Høvsøre. The measurements are represented as median (markers), and first and third quartiles (error bars), respectively. We infer the following:

- The systematic errors vary considerably under different atmospheric stability conditions: the variation is up to $50 \%$ for $u$ and $v$ variances, and up to $20 \%$ for $w$ variance. This is due to a large variation in the length scales of different velocity components resulting in varying attenuation of the variances.

- The systematic errors increase with height under all atmospheric stability conditions; this is due to a quadratic increase in the sample volume with height (Lindelöw 2007). The diameter of the scanning circle also increases with height.

- The systematic errors in $w$ variance are much larger (approximately 3-5 times) than that of the $u$ and $v$ variances; this is due to the very small length scales of the $w$ component as compared to those for $u$ and $v$, resulting in the attenuation of the $w$ variance of up to $90 \%$. The $u$ and $v$ variances are attenuated up to $70 \%$.

- There is a significant spread (first and third quartiles) in the systematic errors of $u$ and $v$ variances; these are the random errors and most likely occur because of the disjunctive sampling (Lenschow et al. 1994) of the ZephIR. 
A thorough scientific investigation is needed to quantify random errors, but is not the focus of this paper.

- The trend of the systematic errors predicted by both models is in agreement with the observations at all heights.

- With the exception of very stable conditions, the model with the low-pass filter [Eqs. (23)-(25)] is in better agreement with the measurements at all heights than without the low-pass filter.

To quantify the improvement in the model predictions using the low-pass filter, we compute the root-meansquare percent errors (RMSPE) between the measured and the modeled systematic errors for each stability condition. RMSPE is given as

$$
\text { RMSPE }=\sqrt{\frac{1}{n} \sum\left[\frac{\left(\frac{\left\langle\mathrm{v}_{i}^{\prime} \mathrm{v}_{i}^{\prime}\right\rangle_{\text {lidar }}}{\left\langle\mathrm{v}_{i}^{\prime} \mathrm{v}_{i}^{\prime}\right\rangle}\right)_{\text {measured }}-\left(\frac{\left\langle\mathrm{v}_{i}^{\prime} \mathrm{v}_{i}^{\prime}\right\rangle_{\text {lidar }}}{\left\langle\mathrm{v}_{i}^{\prime} \mathrm{v}_{i}^{\prime}\right\rangle}\right)_{\text {modelled }}}{\left(\frac{\left\langle\mathrm{v}_{i}^{\prime} \mathrm{v}_{i}^{\prime}\right\rangle_{\text {lidar }}}{\left\langle\mathrm{v}_{i}^{\prime} \mathrm{v}_{i}^{\prime}\right\rangle}\right)_{\text {measured }}}\right]^{2}} \times 100,
$$

where median values are used for the measurements.

Figure 6 shows the comparison of the RMSPE in the prediction of the systematic errors with and without the low-pass filter for the ZephIR. A significant decrease in the RMSPE (of the order of $30 \%$ ) of $u$ and $w$ variances is observed under all atmospheric stabilities (except for the very stable condition for $u$ variance) when the lowpass filtering is used. For the $v$ variance, there is a slight increase (up to $10 \%$ ) in the RMSPE under unstable conditions, whereas for stable conditions a decrease of up to $40 \%$ is observed. Thus, in general, using the lowpass filter, the model predicts the systematic errors better than without using the low-pass filter. We also performed the calculations using the beam radius $r_{b}=$ $24 \mathrm{~mm}$, and observed that the RMSPE for all three variance components changes only slightly $( \pm 5 \%)$.

\section{c. Comparison with the WindCube measurements}

Figure 7 shows the comparison of the modeled and measured systematic errors (section 2a) for $u, v$, and $w$ variances over 10-min periods. We infer the following:

- The systematic errors vary considerably under different atmospheric stability conditions: the variation is up to $50 \%$ for $u$ and $v$ variances, and up to $20 \%$ for the $w$ variance. The same is also observed for the ZephIR.

- The systematic errors decrease with height for the $u$ and $v$ variances under all atmospheric stability conditions: for the WindCube, the probe length is constant (Lindelöw 2007), and, hence, at lower heights there is a combined averaging effect resulting from the probe length and the diameter of the scanning circle. Considering that at lower heights the length scales are smaller than at higher heights, it is likely that the variances are attenuated greater at lower heights than at higher heights. For $w$ variance, the systematic error is approximately constant, and is most likely due to the small length scales.
- The systematic error in $w$ variance is much larger (approximately 3-5 times) than that of the $u$ and $v$ variances. The same is also observed for the ZephIR.

- The spread in the systematic error (first and third quartiles) of the $u$ and $v$ variances is smaller than that of the ZephIR; this is most likely because the WindCube updates the velocity vector approximately every $6.5 \mathrm{~s}$, whereas the ZephIR updates every $18 \mathrm{~s}$.

- The systematic error varies significantly with the wind direction relative to the beam direction for $w$ variance, and to a lesser degree for $u$ and $v$ variance under all stability conditions.

Figure 8 shows the comparison of the RMSPE in the prediction of the systematic errors for the WindCube and ZephIR (with the low-pass filter). It is observed that for $u$ and $v$ variances, with the exception of the nearneutral stable condition, the RMSPE in both lidars is approximately equal. There is a considerable variation in the RMSPE for the $w$ variance. This is most likely because for the WindCube, the $w$ variance is very sensitive to the wind direction because of its cosine and sine dependence. In general, for both lidars, except for the very stable condition, the model predicts the systematic errors for $u$ variance reasonably well (RMSPE $\approx 6 \%$ ), followed by $v$ variance (RMSPE $\approx 12 \%$ ). It is difficult to say whether the prediction for the $w$ variance is less reliable or not (RMSPE of the order of $60 \%)$.

We do not model the filtering effect because of the scanning time $(\approx 6.5 \mathrm{~s})$ of WindCube for two reasons:

(i) Since the measurement is carried out at only four points, with each lasting $0.5 \mathrm{~s}$ on the scanning circle, we cannot assume that the WindCube measures infinitely fast on the scanning circle (as we did for the ZephIR). The translations in each direction have 


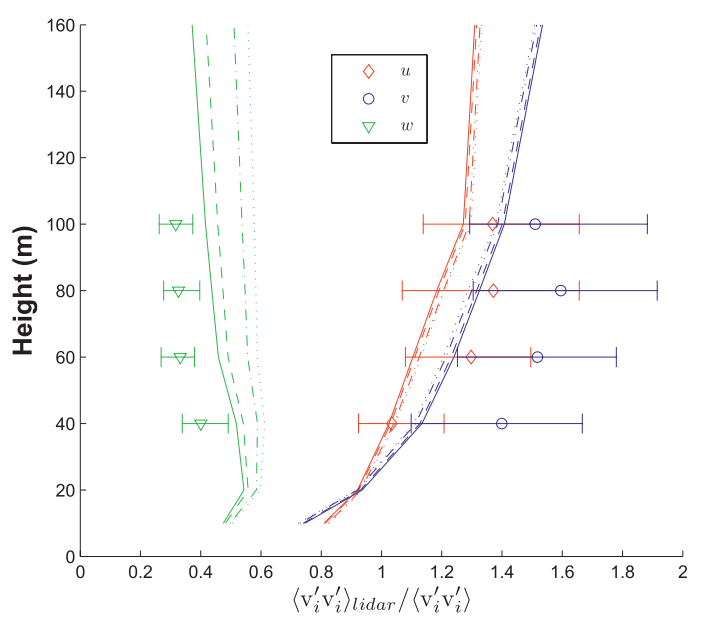

(a) very unstable

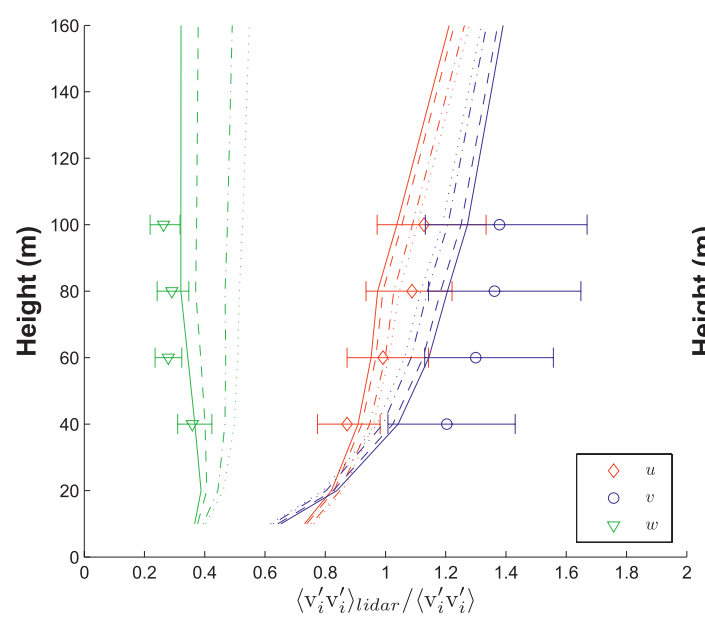

(c) near neutral unstable

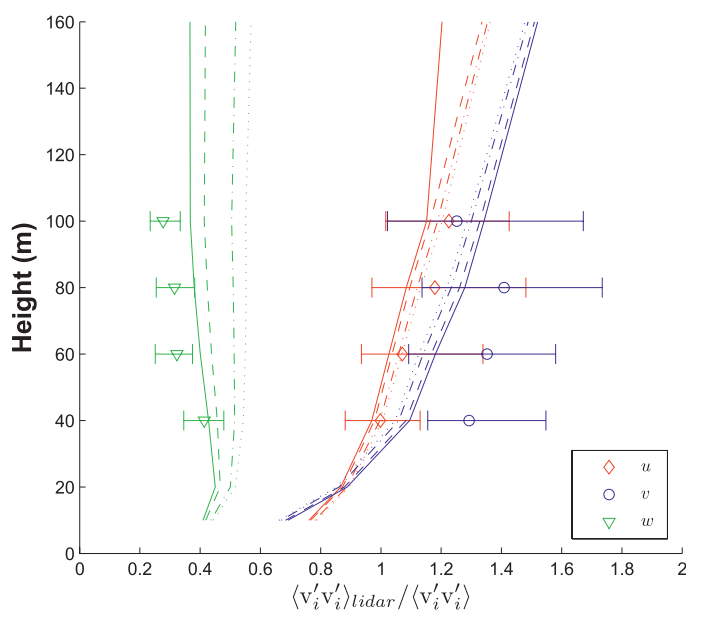

(b) unstable

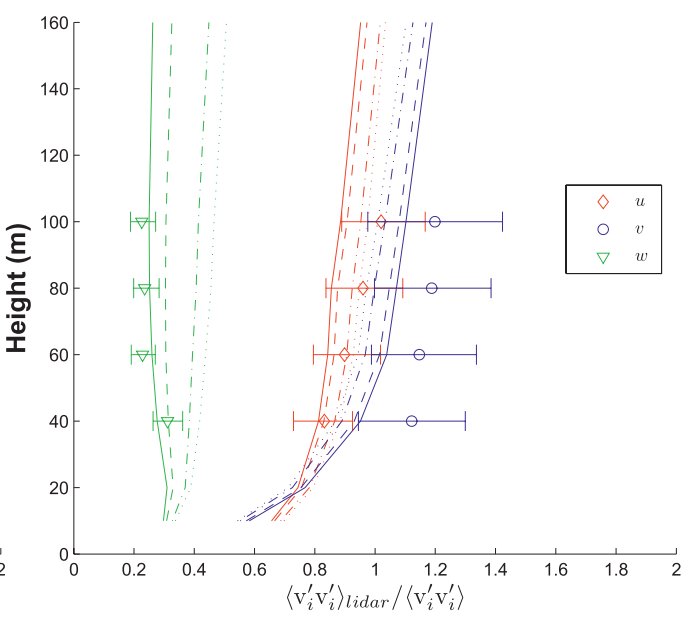

(d) neutral

FIG. 7. As in Fig. 5, but for WindCube systematic errors. Here, the model variation with wind direction is plotted for $0^{\circ}$ (dotted line), $15^{\circ}$ (dash-dot line), $30^{\circ}$ (dashed line), and $45^{\circ}$ (solid line).

to be convolved with the corresponding spectral transfer function, if the filtering is to be included.

(ii) The calculation becomes too cumbersome if the above procedure is followed.

\section{Discussion}

The main goal of this paper is to understand the systematic errors in the second-order moments of $\mathrm{CW}$ and pulsed lidars. In particular, we model the systematic errors for the ZephIR and WindCube, which are used as $\mathrm{CW}$ and pulsed lidars, respectively. Although the model is developed for specific lidars, the modeling framework would be the same for any other instrument. Additionally, we also model the low-pass filter for the 3-s scan in the ZephIR. We expected a large variation in the systematic errors under different atmospheric stability conditions and, hence, performed the analysis accordingly. Figures 5 and 7 indeed justify our analysis.

In general, except for the very stable conditions, the model predicts the systematic errors quite well, where the RMSPE for the $u$ and $v$ variances are of the order of $4 \%$ and $15 \%$, respectively. For the ZephIR, when the low-pass filter is not used then the RMSPE is quite large (of the order of $30 \%$ ) for the $u$ variance. For the $w$ variance, the high values of RMSPE (of the order of $60 \%$ ) under all atmospheric stability conditions are observed. We think that the following two reasons could contribute to this:

(i) The attenuation in the $w$ variance is quite large (up to $90 \%$ ), as compared to the $u$ and $v$ variances (up to $70 \%$ ). Thus, a small difference in the model prediction and the measurements results in amplifying the RMSPE. 


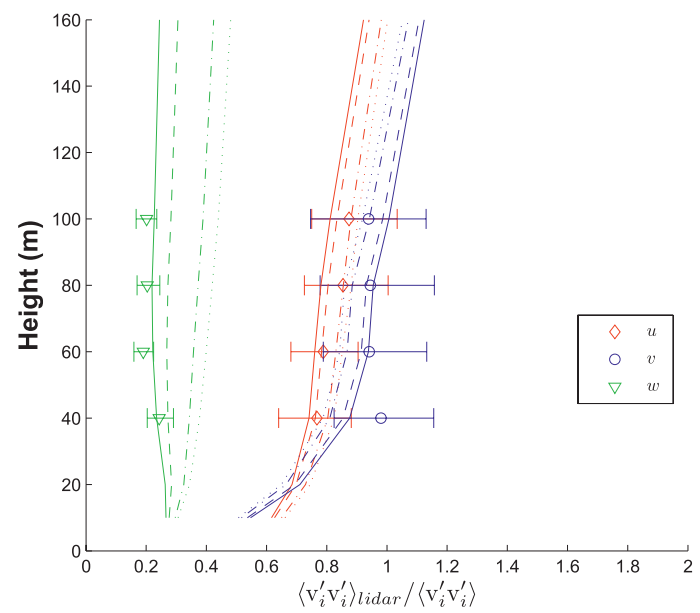

(e) near neutral stable

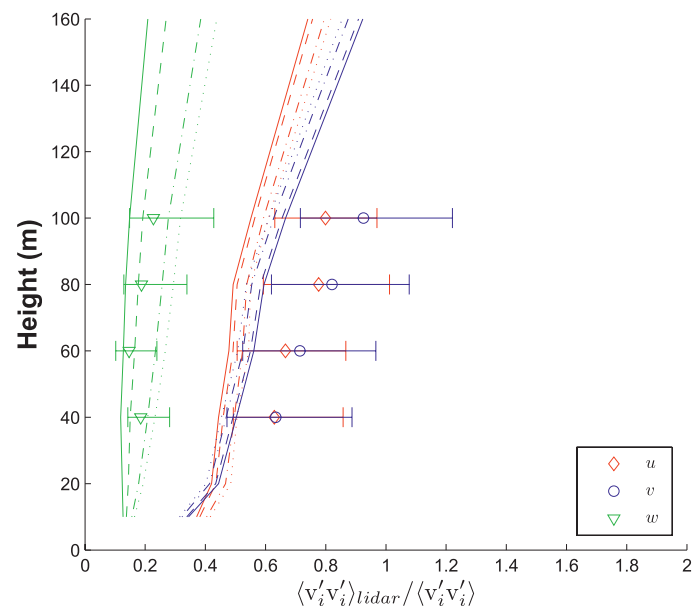

(g) very stable

(ii) For the ZephIR, when the low-pass filter is used in the model, there is a dependence on the mean wind speed. The model results (Fig. 5) are shown for $\langle u\rangle=$ $9 \mathrm{~m} \mathrm{~s}^{-1}$ only. Segregating the model and observations for different mean wind speeds will result in reducing the RMSPE.

For the WindCube, the model predicts a significant variation of the $w$ variance with wind direction [Eq. (30)]. To estimate the influence of the weights $P$ and $Q$ on the prediction of systematic errors, we calculate $\left\langle w_{\mathrm{wc}}^{\prime 2}\right\rangle$ from the equation for $w$ that corresponds to Eq. (38) with two different ways of calculating $w$. The first is the formula used by Leosphere, for example, Eq. (30), with $P=\cos ^{2} \Theta$ and $Q=\sin ^{2} \Theta$; the second is $P=Q=1 / 2$. The former is shown as a thin solid line in Fig. 9 and the latter as a thin dashed line. The spectral tensor parameters used are for neutral atmospheric stability from Peña et al. (2010) at $100 \mathrm{~m}$. The measurements of $\left\langle w_{\mathrm{wc}}^{\prime 2}\right\rangle /\left\langle w^{\prime 2}\right\rangle$, shown as broad curves on Fig. 9, are from the same height, and both

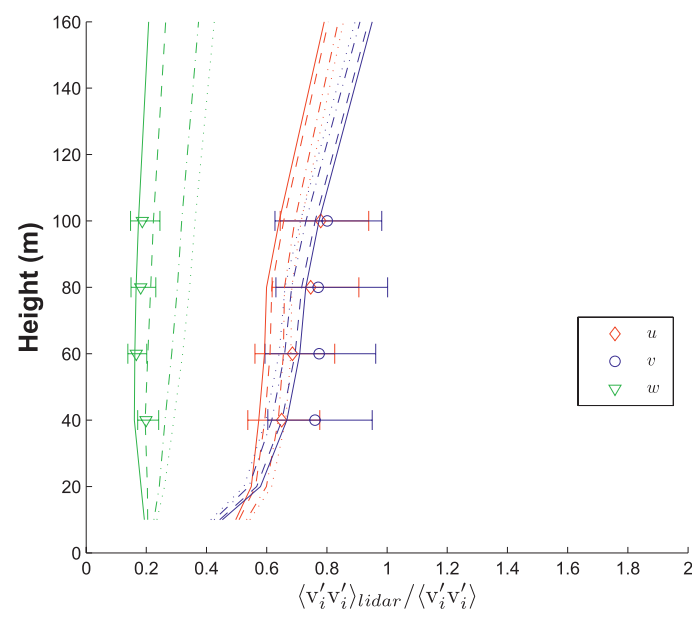

(f) stable

FIG. 7. (Continued)

measurements and theory show that $\left\langle w_{\mathrm{wc}}^{\prime 2}\right\rangle /\left\langle w^{\prime 2}\right\rangle$ using Leosphere's choice of $P$ and $Q$ can vary by a factor of 2 solely by changing the wind direction. If $P=Q=1 / 2$ is chosen, then the reduction of the vertical velocity variance does vary much less with wind direction, but the overall attenuation is stronger.

Because the model predicts the trend in the systematic errors in the $w$ variance reasonably well (Figs. 5 and 7), qualitatively it could be said that the model also agrees well with the measurements for the $w$ variance.

While comparing the performance of our model, the following should also be considered:

- The model is dependent on the three-dimensional spectral velocity tensor (Mann 1994), which is strictly valid for neutral conditions only. Thus, one has to be careful while comparing under different atmospheric stability conditions. In this study, we have reduced the uncertainty by using the three input tensor parameters that are fitted to the measurements under 


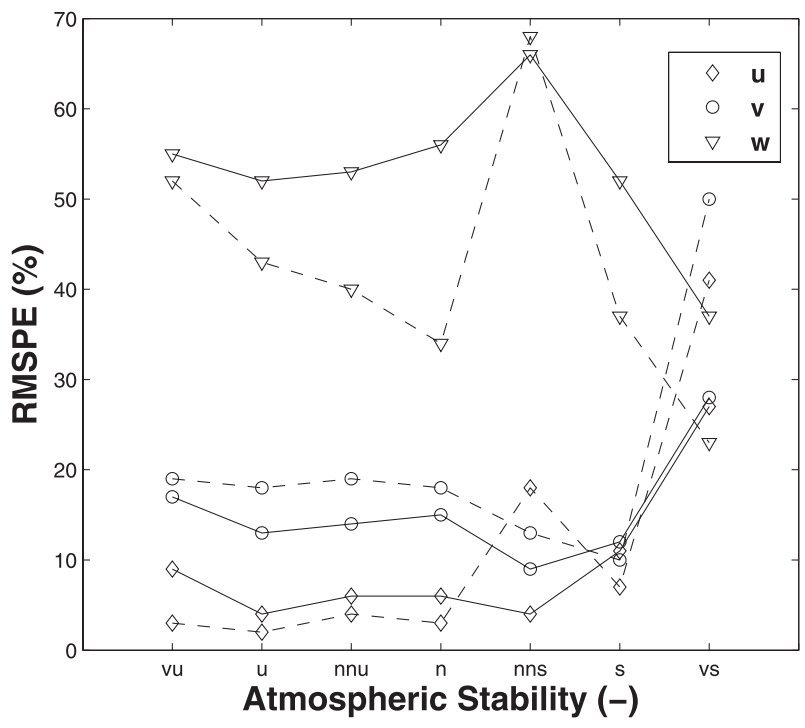

FIG. 8. Comparison of the RMSPE in the prediction of the systematic errors for the WindCube and ZephIR. The WindCube (solid line) and ZephIR (dashed line) with the low-pass filter are shown. See Table 1 for the meaning of the abbreviations on the $x$ axis.

different atmospheric stability conditions (Peña et al. 2010).

- While using Eqs. (23)-(25), we have used the same mean wind speed at all heights. In reality, there is always wind shear, which also depends significantly on atmospheric stability (Motta and Barthelmie 2005). However, the calculations will become too cumbersome, and hence we made a crude approximation.

- The very stable conditions are generally difficult to analyze. There could be different reasons for the large deviation in the $u$ and $v$ variances; for example, uncertainty in the input tensor parameters, and lack of validity of the spectral tensor model (Mann 1994) under different atmospheric stability conditions

- Also, contrary to expectation, the measurements under very stable conditions (Figs. 5 and 8) show a decrease in the systematic errors for the $u$ and $v$ variances, as compared to the stable conditions.

There is also some room for reducing redundancy in the ZephIR measurements, which might reduce the spread of the systematic errors (quartile range). Instead of scanning at several points on the circle, only four points are required. Reducing the measurement points would increase the dependence of the second-order moments on the wind direction (cf. section 2). However, it would considerably reduce the time required for completing a VAD. There is also no need to scan the circle 3 times; for example, in the present configuration, 50 points are scanned in approximately $1 \mathrm{~s}$. Thus, four points would take only $0.08 \mathrm{~s}$. If it measures five heights sequentially,

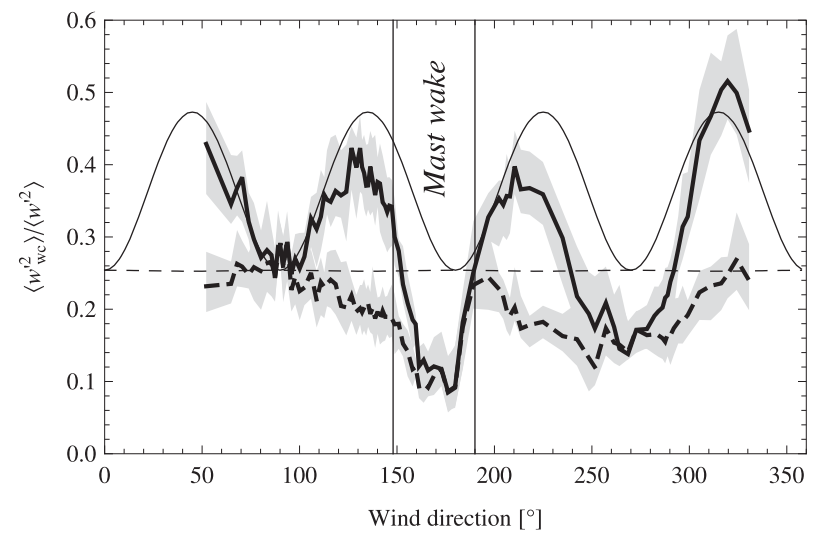

FIG. 9. The ratio of the vertical velocity variance as measured by the WindCube and the actual variance measured and modeled at $100 \mathrm{~m}$. The theoretical expectations (thin lines) using $P=\cos ^{2} \Theta$ and $Q=\sin ^{2} \Theta$ (solid line) and $P=Q=1 / 2$ (dashed line) in Eq. (30) are shown. The corresponding measurements are shown (broad curves), with the first and third quartiles displayed (shades).

then the next measurement would be after $0.4 \mathrm{~s}$, giving a measurement frequency of $\gtrsim 2 \mathrm{~Hz}$. Alternatively, at each of the four points the scans can also be performed rapidly at different heights sequentially before scanning the next point.

We are currently looking into alternative ways of analyzing the lidar data and different beam configurations that would render turbulence measurements more feasible. One idea is to use two different half opening angles as in Eberhard et al. (1989), who show that all terms in the Reynolds stress tensor can be obtained by using the single-beam statistics, without resorting to beam covariances, which is done in this paper. That would require significant hardware modifications to the instruments treated here. Another idea is to supplement the analysis with information on the width of the Doppler spectra, as done for the momentum flux in Mann et al. (2010), in order to compensate for the effect of alongbeam averaging.

\section{Conclusions}

The systematic errors of the second-order moments measured by lidars using the conical scanning and VAD technique to process the data are quite large because of

(i) the spatial separation of the data points along the line-of-sight and

(ii) the spatial separation of the data points in the conical section.

Also, from Eqs. (14), (17), (18), and (43), the general lidar equation for the second-order moments using the VAD data processing technique can be written as 


$$
\begin{aligned}
\left\langle\mathrm{v}_{m}^{\prime} \mathrm{v}_{n}^{\prime}\right\rangle_{\mathrm{lidar}} & =\int \Phi_{i j}(\mathbf{k}) X_{i}^{m}(\mathbf{k}) X_{j}^{n *}(\mathbf{k}) d \mathbf{k} ; \\
X_{i}^{m}(\mathbf{k}) & =\left\{\begin{array}{cl}
\beta_{i}(\mathbf{k}) \wedge b_{i}(\mathbf{k}), & m=1 \\
\gamma_{i}(\mathbf{k}) \wedge c_{i}(\mathbf{k}), & m=2 . \\
\alpha_{i}(\mathbf{k}) \wedge a_{i}(\mathbf{k}), & m=3
\end{array}\right.
\end{aligned}
$$

The weighting functions $\alpha_{i}(\mathbf{k}), \beta_{i}(\mathbf{k})$, and $\gamma_{i}(\mathbf{k})$ are used for the ZephIR and $a_{i}(\mathbf{k}), b_{i}(\mathbf{k})$, and $c_{i}(\mathbf{k})$ are used for the WindCube. Thus, the measurement of the second-order moment by lidar involves interaction of all components of the spectral velocity tensor $\Phi_{i j}(\mathbf{k})$ weighted by the corresponding weighting functions $X_{i}^{m}(\mathbf{k})$. It is to be noted that Eq. (48) is given in Einstein summation convention, and, hence, in order to explicitly see the contribution of all components of $\Phi_{i j}(\mathbf{k})$ on the measurement of the second-order moments by lidar, this equation must be expanded for all values of the subscripts $i$ and $j$. In most cases, this results in the attenuation of the second-order moments, whereas in some cases this also results in amplification of the secondorder moment, for example, as observed for the WindCube in the unstable conditions (see Fig. 7).

Finally, to answer the question posed in the title "Can wind lidars measure turbulence?", it is clear that using the conical scanning and VAD technique to process the data they cannot be used to measure turbulence precisely.

Acknowledgments. This study is a part of the Ph.D. project under the We@Sea program, BISK-03041, and sponsored by the Dutch Ministry of Economic affairs. The experimental results are all based on data collected at the Høvsøre Test Station under the watchful eyes of Anders Ramsing Vestergaard and Bjarne Sønderskov. We extend our thanks to them for their highly professional and good-humored assistance. We are also obliged to the NORSEWInD project and its leader Andy Oldroyd, under whose auspices the lidar data were obtained. Finally, we are grateful for valuable comments from Torben Mikkelsen, Ris $\varnothing$ DTU. This paper has been prepared using resources provided by the EU FP6 UpWind project (Project reference 019945 SES6) and by the Center for Computational Wind Turbine Aerodynamics and Atmospheric Turbulence funded by the Danish Council for Strategic Research Grant 09-067216.

\section{REFERENCES}

Banakh, V. A., I. N. Smalikho, F. Köpp, and C. Werner, 1995: Representativeness of wind measurements with a CW Doppler lidar in the atmospheric boundary layer. Appl. Opt., 34, 20552067.

Banta, R. M., R. K. Newsom, J. K. Lundquist, Y. L. Pichugina, R. L. Coulter, and L. Mahrt, 2002: Nocturnal low-level jet characteristics over Kansas during CASES-99. Bound.-Layer Meteor., 105, 221-252.

Browning, K. A., and R. Wexler, 1968: The determination of kinematic properties of a wind field using a Doppler radar. J. Appl. Meteor., 7, 105-113.

Citriniti, J. H., and W. K. George, 1997: The reduction of spatial aliasing by long hot-wire anemometer probes. Exp. Fluids, 23, 217-224.

Courtney, M., R. Wagner, and P. Lindelow, 2008: Testing and comparison of lidars for profile and turbulence measurements in wind energy. Proc. 14th Int. Symp. for the Advancement of Boundary Layer Remote Sensing, Vol. 1, Risø DTU, Denmark, IOP Conference Series: Earth and Environmental Science, 012021.

Eberhard, W. L., R. E. Cupp, and K. R. Healy, 1989: Doppler lidar measurements of profiles of turbulence and momentum flux. J. Atmos. Oceanic Technol., 6, 809-819.

Emeis, S., M. Harris, and R. M. Banta, 2007: Boundary-layer anemometry by optical remote sensing for wind energy applications. Meteor. Z., 16, 337-347, doi:10.1127/0941-2948/2007/0225.

Engelbart, D. A. M., M. Kallistratova, and R. Kouznetsov, 2007: Determination of the turbulent fluxes of heat and momentum in the ABL by ground-based remote-sensing techniques (a review). Meteor. Z., 16, 325-335, doi:10.1127/0941-2948/2007/ 0224.

Gal-Chen, T., M. Xu, and W. L. Eberhard, 1992: Estimation of atmospheric boundary layer fluxes and other turbulence parameters from Doppler lidar data. J. Geophys. Res., 97 (D17), 18 409-18 423.

Genz, A. C., and A. A. Malik, 1980: An adaptive algorithm for numerical integration over an n-dimensional rectangular region. J. Comput. Appl. Math., 6 (4), 295-302.

Gryning, S.-E., E. Batchvarova, B. Brümmer, H. Jørgensen, and S. Larsen, 2007: On the extension of the wind profile over homogeneous terrain beyond the surface layer. Bound.-Layer Meteor., 124, 251-268, doi:10.1007/s10546-007-9166-9.

IEC, 2005a: Wind turbines-Part I: Design requirements. International Electrotechnical Commission IEC 61400-1, 92 pp. , 2005b: Offshore wind turbines-Part I: Design requirements. International Electrotechnical Commission IEC 61400-3, 136 pp.

— 2005c: Wind turbines-Part 12-1: Power performance measurements of electricity producing wind turbines. International Electrotechnical Commission IEC 61400-12-1, 90 pp.

Kaimal, J. C., and J. J. Finnigan, 1994: Acquisition and processing of atmospheric boundary layer data. Atmospheric Boundary Layer Flows, Oxford University Press, 255-257.

Kindler, D., A. Oldroyd, A. Macaskill, and D. Finch, 2007: An eight month test campaign of the QinetiQ ZephIR system: Preliminary results. Meteor. Z., 16 (5), 479-489, doi:10.1127/ 0941-2948/2007/0226.

Kropfli, R. A., 1986: Single Doppler radar measurement of turbulence profiles in the convective boundary layer. J. Atmos. Oceanic Technol., 3, 305-314.

Lenschow, D. H., J. Mann, and L. Kristensen, 1994: How long is long enough when measuring fluxes and other turbulence statistics? J. Atmos. Oceanic Technol., 11, 661-673.

Lindelöw, P., 2007: Fibre based coherent lidars for remote wind sensing. Ph.D. thesis, Technical University Denmark, 164 pp.

Lindelöw-Marsden, P., 2007: UpWind D1: Uncertainties in wind assessment with LIDAR. Ris $\varnothing$ DTU Tech. Rep. Ris $\varnothing-R-1681(E N)$, $55 \mathrm{pp}$.

Mann, J., 1994: The spatial structure of neutral atmospheric surface-layer turbulence. J. Fluid Mech., 273, 141-168. 
A. Pena, F. Bingöl, R. Wagner, and M. S. Courtney, 2010: Lidar scanning of momentum flux in and above the surface layer. J. Atmos. Oceanic Technol., 27, 792-806.

, and Coauthors, 2009: Comparison of 3D turbulence measurements using three staring wind lidars and a sonic anemometer. Meteor. Z., 18 (2), 135-140, doi:10.1127/0941-2948/ 2009/0370

Monin, A. S., and A. M. Yaglom, 1975: Statistical Fluid Mechanics. Vol. 2. MIT Press, 886 pp.

Motta, M., and R. J. Barthelmie, 2005: The influence of nonlogarithmic wind speed profiles on potential power output at Danish offshore sites. Wind Energy, 8, 219-236.

Peña, A., S.-E. Gryning, and J. Mann, 2010: On the length scale of the wind profile. Quart. J. Roy. Meteor. Soc., 136 (653), 2119-2131.

, C. B. Hasager, S.-E. Gryning, M. Courtney, I. Antoniou, and T. Mikkelsen, 2009: Offshore wind profiling using light detection and ranging measurements. Wind Energy, 12, 105-124, doi:10.1002/we.283.

Pichugina, Y. L., R. M. Banta, N. D. Kelly, B. J. Jonkman, S. C. Tucker, R. K. Newsom, and W. A. Brewer, 2008: Horizontalvelocity and variance measurements in the stable boundary layer using Doppler lidar: Sensitivity to averaging procedures. J. Atmos. Oceanic Technol., 25, 1307-1327.
Sjöholm, M., T. Mikkelsen, J. Mann, K. Enevoldsen, and M. Courtney, 2009: Spatial averaging-effects on turbulence measured by a continuous-wave coherent lidar. Meteor. Z., 18 (3), 281-287, doi:10.1127/0941-2948/2009/0379.

Smalikho, I., F. Kopp, and S. Rahm, 2005: Measurement of atmospheric turbulence by $2-\mu \mathrm{m}$ Doppler lidar. J. Atmos. Oceanic Technol., 22, 1733-1747.

Smith, D. A., M. Harris, A. S. Coffey, T. Mikkelsen, H. E. Jørgensen, J. Mann, and R. Danielian, 2006: Wind lidar evaluation at the Danish wind test site in Høvsøre. Wind Energy, 9, 87-93, doi:10.1002/we.193.

Sonnenschein, C. M., and F. A. Horrigan, 1971: Signal-to-noise relationships for coaxial systems that heterodyne backscatter from atmosphere. Appl. Opt., 10, 1600-1604.

Wagner, R., T. Mikkelsen, and M. Courtney, 2009: Investigation of turbulence measurements with a continuous wave, conically scanning lidar. Ris $\varnothing$ DTU Tech. Rep. Ris $\varnothing-R-1682(E N)$, $22 \mathrm{pp}$.

Wilson, D. A., 1970: Doppler radar studies of boundary layer wind profiles and turbulence in snow conditions. Proc. 14th Conf. on Radar Meteorology, Tucson, AZ, Amer. Meteor. Soc., 191-196.

Wyngaard, J. C., 1968: Measurement of small-scale turbulence structure with hot wires. J. Sci. Instrum., 1, 1105-1108. 